\title{
High-quality-factor AlGaAs optical microcavities for atomic Cs and semiconductor quantum dot cavity QED experiments
}

\author{
Kartik Srinivasan, Paul E. Barclay, and Oskar Painter \\ Thomas J. Watson, Sr: Laboratory of Applied Physics, California Institute of Technology, Pasadena, CA 91125, USA. \\ phone: (626) 395-6269, fax: (626) 795-7258, e-mail: kartik@caltech.edu \\ Andreas Stintz and Sanjay Krishna \\ Center for High Technology Materials, University of New Mexico, Albuquerque, NM 87106, USA.
}

\begin{abstract}
High-quality-factor $\left(Q \sim 10^{4}-10^{5}\right) \mathrm{AlGaAs}$ microdisks for atomic and semiconductor quantum dot cavity QED experiments are demonstrated. Cavities embedded with InAs/InGaAs dots-in-a-well exhibit room-temperature pulsed lasing with thresholds $<17 \mathrm{uW}$. Continuous wave operation at roomtemperature is also observed. (c) 2005 Optical Society of America

OCIS codes: (230.5750) Resonators; (270.0270) Quantum Optics; (140.5960) Semiconductor Lasers
\end{abstract}

Within the past year, demonstrations of high quality factor $(Q)$, small mode volume $\left(V_{\text {eff }}\right)$ silicon photonic crystal microcavities[1, 2] $\left(Q \sim 4 \times 10^{4}, V_{\mathrm{eff}} \sim(\lambda / n)^{3}\right)$ and microdisks[3] $\left(Q \sim 5 \times 10^{5}, V_{\mathrm{eff}} \sim 5(\lambda / n)^{3}\right)$ have indicated the potential of semiconductor microcavities for chip-based quantum optics experiments, such as the recent demonstrations of vacuum Rabi splitting in a microcavity-quantum dot system[4, 5]. Experiments integrating such microcavities with chip-level magnetic microtraps to deliver cooled Cs atoms to the cavity near-field for experiments within the strong coupling domain of cavity quantum electrodynamics (cQED) have been proposed[6]. However, the Cs D2 transition commonly used in cQED experiments is at a wavelength $\lambda \sim 852 \mathrm{~nm}$, where silicon is highly absorbing. The bandgap of AlGaAs, on the other hand, can be tailored (as a function of Al content) so that it is transparent in this wavelength region, and is thus a more suitable choice for such experiments. Furthermore, AlGaAs has the advantage over silicon (and other materials such as silica, where ultra-high-Q, larger $V_{\text {eff }}$ microtoroid cavities have been demonstrated[7]) in that quantum-confined structures such as quantum wells and dots can be incorporated during its epitaxial growth, allowing for purely semiconductor cQED experiments $[4,5]$.

Here, we report on recent progress in creating high- $Q$ AlGaAs microdisks in the $\sim 850 \mathrm{~nm}\left(Q \sim 3.4 \times 10^{4}\right)$ and $1550 \mathrm{~nm}\left(Q \sim 1.5 \times 10^{5}\right)$ wavelength bands, which compare favorably with the highest $Q$ factors measured for AlGaAs microcavities $[8,9,4,5]$. The utility of such cavities has been demonstrated in their integration with quantum dots-ina-well (DWELL) epitaxies[10,11] to create optically pumped, low threshold, room temperature quantum dot lasers at $\lambda \sim 1.2 \mu \mathrm{m}$. Laser thresholds of $<17 \mu \mathrm{W}$ of peak external pump power have been achieved in disks containing a single DWELL layer, representing over an order of magnitude decrease in comparison to recent experimental results[12]. The loss in these cavities is low enough that the lasers operate at thresholds approaching the transparency levels of the DWELL material, previously only seen in broad-area stripe lasers with mm-scale cavity lengths[11].

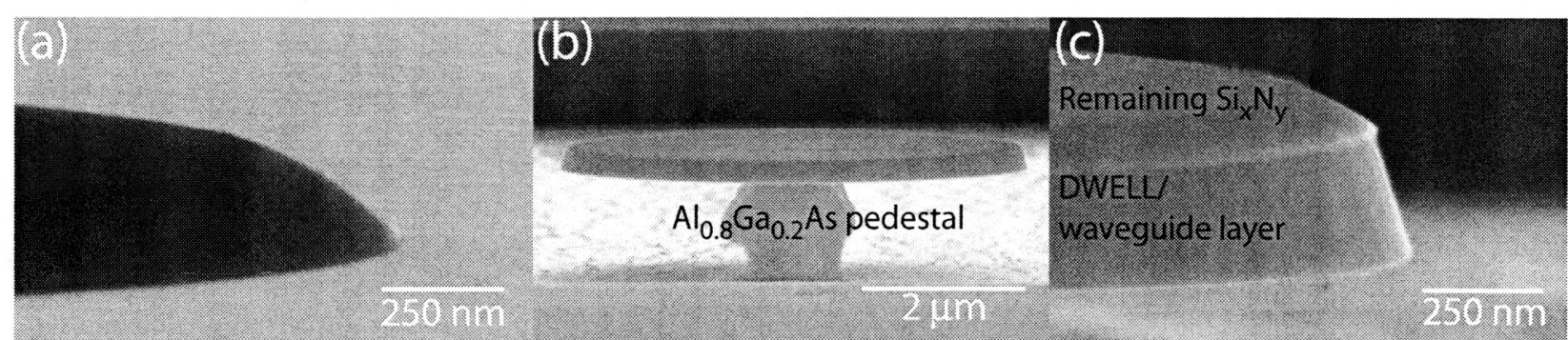

Fig. 1. Scanning electron microscope (SEM) images of microdisk cavities after the (a) $\mathrm{Sj}_{\mathbf{j}} \mathrm{N}_{y}$ etch, and (b)-(c) AlGaAs etch and undercut.

Initial passive $Q$ measurements are performed on $d=6 \mu$ m diameter microdisk cavities fabricated in a $180 \mathrm{~nm}$ thick $\mathrm{Al}_{0.3} \mathrm{Ga}_{0.7}$ As layer supported by a $1.5 \mu \mathrm{m}$ thick $\mathrm{Al}_{0.8} \mathrm{Ga}_{0.2} \mathrm{As}$ pedestal (Fig. 1). The cavities are created through: (i) electron beam lithography and subsequent reflow of the resist, (ii) $\mathrm{SF}_{6} / \mathrm{C}_{4} \mathrm{~F}_{8}$ inductively-coupled plasma reactive ion etching (ICP-RIE) of a deposited $\mathrm{Si}_{x} \mathrm{~N}_{y}$ mask layer (Fig. 1(a)), (iii) $\mathrm{Ar}-\mathrm{Cl}_{2}$ ICP-RIE etching of the $\mathrm{Al}_{0.3} \mathrm{Ga}_{0.7} \mathrm{As}$ layer, and (iv) wet chemical etching of the underlying $\mathrm{Al}_{0.8} \mathrm{Ga}_{0.2}$ As layer to form the supporting pedestal (Fig. 1(b)-(c)). The $\mathrm{Si}_{x} \mathrm{~N}_{y}$ etch step is particularly important, as any roughness in this mask layer is transferred into the underlying layers. 


\section{QME5}

Srinivasan, et al., High quality factor AlGaAs optical microcavities...

QELS/2005 Page 2

This etch has thus been calibrated to produce as smooth a sidewall surface as possible (Fig. 1(a)), without particular concern for the verticality of the etched sidewall. The subsequent $\mathrm{Ar}-\mathrm{Cl}_{2}$ etch is highly selective so that the angled mask does not result in erosion of the AlGaAs sidewalls.

The $Q$ factors of the cavity modes are directly probed using an optical-fiber-based evanescent coupling approach $[2$, 3]. In Fig. 2(a), we show the resonance of a microdisk mode in the $850 \mathrm{~nm}$ wavelength band. The linewidth of this resonance $(\gamma)$, determined by a Lorentzian fit to the data, corresponds to $Q \sim 3.4 \times 10^{4}$, and is a minimum estimate for the $Q$, as loading of the cavity due to the presence of the taper causes $\gamma$ to broaden past its cold-cavity value. Similarly, in Fig. 2(b), we show a wavelength scan for one of the disks in the $1550 \mathrm{~nm}$ band. In this scan, we see a number of resonances with $Q \mathrm{~s}$ as high as $1.5 \times 10^{5}$. We believe that the lower $Q$ factors in the $850 \mathrm{~nm}$ band might be due to incomplete removal of a GaAs cap layer, which will be much more strongly absorbing at lower wavelengths. Further improvements in this step, along with etched sidewalls whose roughness is comparable to those in the high- $Q$ Si microdisks[3] indicate that $Q>10^{5}$ at $\sim 850 \mathrm{~nm}$ should be achievable.

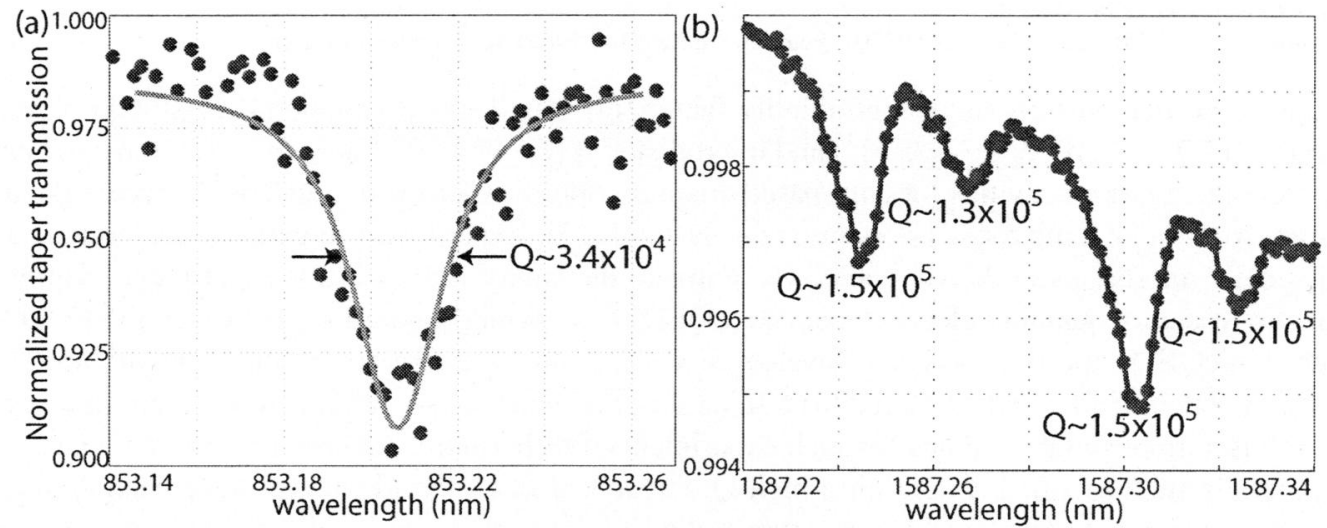

Fig. 2. (a) Normalized taper transmission for a $6 \mu \mathrm{m}$ diameter microdisk in the $850 \mathrm{~nm}$ range. (b) Normalized taper transmission for a $6 \mu \mathrm{m}$ diameter microdisk in the $1550 \mathrm{~nm}$ range.

The InAs/InGaAs DWELL system has proven to be a technologically important system due to its near-IR emission wavelengths which approach the zero dispersion region of optical fibers as well as their superior performance in very low threshold laser devices [11]. The epitaxy used here consists of one or three layers of InAs quantum dots embedded in (1-3) $\mathrm{In}_{0.15} \mathrm{Ga}_{0.85}$ As quantum wells, which are in turn sandwiched between $\mathrm{GaAs} / \mathrm{Al}_{0.3} \mathrm{Ga}_{0.7}$ As layers to create a total waveguide layer that is $255 \mathrm{~nm}$ thick. This layer is grown on top of a $1.5 \mu \mathrm{m}$ thick $\mathrm{Al}_{0.8} \mathrm{Ga}_{0.2} \mathrm{As}$ sacrificial layer, and the disks are fabricated using the process described earlier. Cavities $(d=6 \mu \mathrm{m})$ are optically pumped at room temperature using a pulsed $830 \mathrm{~nm}$ semiconductor laser, and the emitted laser light at $\sim 1.2 \mu \mathrm{m}$ is collected by a microscope objective and resolved by an optical spectrum analyzer (OSA). For both the 1- and 3-DWELL devices, lasing action is observed for a few ( 2-5) modes in a given microdisk, and the linewidth of the resonant modes (taken at sub-threshold pump powers) are as narrow as the resolution limit of the OSA (inset of Fig. 3(a)). Fig. 3(a) shows a typical light-in-light-out (L-L) curve for a 3-DWELL device pumped with a $300 \mathrm{~ns}$ period and $10 \mathrm{~ns}$ pulse width; the estimated threshold value from this curve is $\sim 22 \mu \mathrm{W}$. Similarly, Fig. 3(b) shows an L-L curve for a 1-DWELL device. This material's ground state saturated modal gain is estimated to be $3.6 \mathrm{~cm}^{-1}$ [11], indicating that a minimum cavity $Q \sim 4 \times 10^{4}$ is required for this single layer of dots to provide enough gain compensation just to achieve lasing. Lasing from the quantum dot ground states is indeed observed, and furthermore, the threshold of $16.4 \mu \mathrm{W}$ (Fig. 3(b)) is significantly lower than the $750 \mu \mathrm{W}$ threshold value recently reported for similarly sized microdisk quantum dot lasers[12]. The thresholds we report are the peak pump power incident on the sample surface; the absorbed pump power is conservatively estimated to be roughly $16 \%$ of this value. The equivalent threshold current density, useful for comparing their performance to previously demonstrated broad area stripe lasers[11], can be estimated by knowing the pump spot size $\left(\sim 16 \mu \mathrm{m}^{2}\right)$ and assuming an internal quantum efficiency $\sim 1$. From this calculation, we arrive at estimated threshold current densities of $\sim 15$ and $11 \mathrm{~A} / \mathrm{cm}^{2}$ for the 3- and 1-DWELL devices, respectively. In comparison, the estimated transparency current densities in previous work are 20.1 and $10.1 \mathrm{~A} / \mathrm{cm}^{2}$, respectively[11]. The proximity of the demonstrated laser thresholds to these transparency values is due to the high cavity $Q$ factors that have largely reduced non-intrinsic losses from the system. Finally, we note that room temperature, continuous wave (CW) lasing was observed for both 3- and 1-DWELL lasers (Fig. 3(c)), albeit at somewhat higher thresholds. 


\section{QME5}
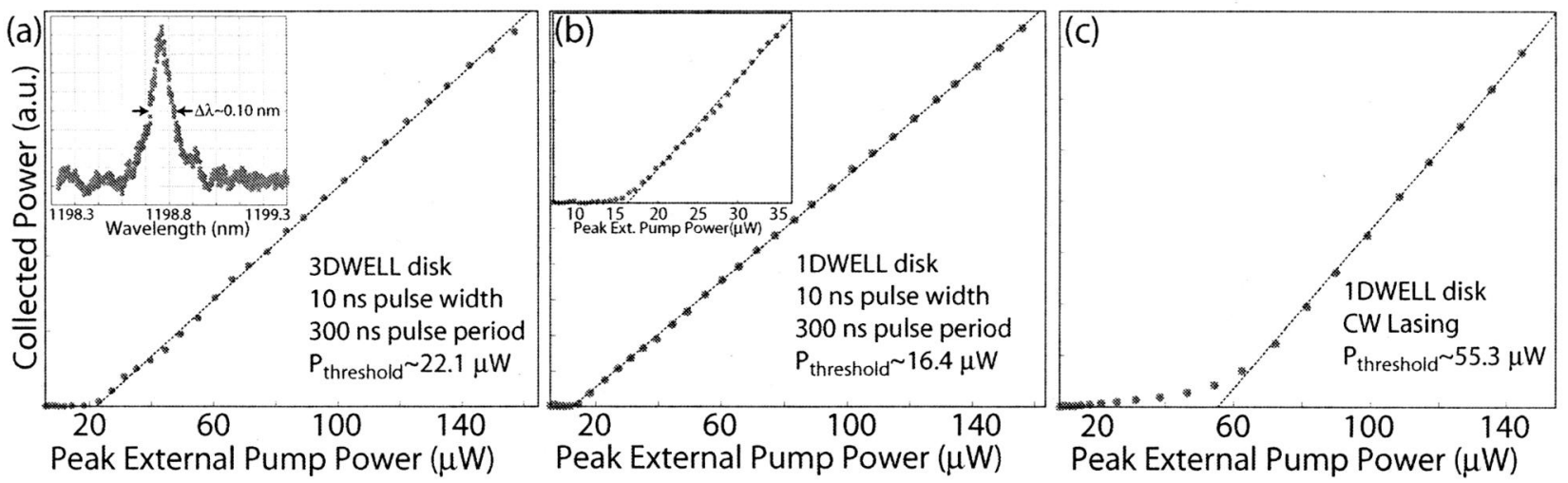

Fig. 3. L-L curves for: (a) 3DWELL microdisk laser. The inset shows the sub-threshold spectrum of cavity mode with a resolution limited linewidth. (b) 1DWELL microdisk laser. The inset shows a zoomed-in near-threshold L-L curve obtained by using an attenuator to achieve higher resolution when incrementing the pump beam power. (c) 1DWELL microdisk laser under $\mathrm{CW}$ pumping conditions.

In addition to the aforementioned results, a number of extensions of this work that are currently in progress will be discussed. These include improvements in the $Q$ of $6 \mu \mathrm{m}$ microdisks at $\lambda \sim 850 \mathrm{~nm}$ and investigations on smaller diameter disks and on the high- $Q$, ultrasmall $V_{\text {eff }}$ photonic crystal cavities previously demonstrated in silicon[2]. Furthermore, the advantage of using the optical-fiber-probing technique in experiments will be highlighted. In particular, the fiber taper can serve not only as a probe to measure $Q$ factors, but also as an input coupler that allows for light to be efficiently transferred from an optical fiber into the wavelength scale mode volume of the cavity, where it can interact with embedded quantum dots or introduced Cs atoms, and as a subsequent output coupler. This technique can greatly improve upon the coupling efficiency to wavelength-scale microcavities such as disks and photonic crystals[13], which typically do not have a radiation pattern that can efficiently be collected by free-space optics or a cleaved fiber[5]. This is of particular importance in quantum optics experiments where the light intensity required for single-photon-based experiments can be quite low, and is integral to recently proposed cQED experiments with cooled Cs atoms[6]. Preliminary applications of this method towards fiber-based pumping and collection schemes for the microdisk DWELL lasers described above will be examined. Finally, initial low temperature $(T<10 \mathrm{~K})$ measurements of the DWELL epitaxy and DWELL-containing microcavities will be discussed.

\section{References}

1. Y. Akahane, T. Asano, B.-S. Song, and S. Noda, "High- $Q$ photonic nanocavity in a two-dimensional photonic crystal," Nature 425 , $944-947$ (2003).

2. K. Srinivasan, P. E. Barclay, M. Borselli, and O. Painter, "Optical-fiber-based measurement of an ultrasmall volume, high- $Q$ photonic crystal microcavity," Phys. Rev. B 70,081206(R) (2004).

3. M. Borselli, K. Srinivasan, P. Barclay, and O. Painter, "Rayleigh scattering, mode coupling, and optical loss in silicon microdisks," Appl. Phys. Lett. 85, 3693-3695 (2004).

4. J. Reithmaier, G. Sek, A. Loffer, C. Hoffman, S. Kuhn, S. Reitzenstein, L. Keldysh, V. Kulakovskii, T. Reinecke, and A. Forchel, "Strong coupling in a single quantum dot-semiconductor microcavity system," Nature 432, 197-200 (2004).

5. T. Yoshie, A. Scherer, J. Hendrickson, G. Khitrova, H. Gibbs, G. Rupper, C. Ell, Q. Schenkin, and D. Deppe, "Vacuum Rabi splitting with a single quantum dot in a photonic crystal nanocavity," Nature 432, 200-203 (2004).

6. B. Lev, K. Srinivasan, P. E. Barclay, O. Painter, and H. Mabuchi, "Feasibility of detecting single atoms with photonic bandgap cavities," Nanotechnology 15, S556-S561 (2004).

7. D. K. Armani, T. J. Kippenberg, S. M. Spillane, and K. J. Vahala, "Ultra-high- $Q$ toroid microcavity on a chip," Nature 421, $925-928$ (2003).

8. B. Gayral, J. M. Gérard, A. Lemaitre, C. Dupuis, L. Manin, and J. L. Pelouard, "High- $Q$ wet-etched GaAs microdisks containing InAs quantum boxes," Appl. Phys. Lett. 75, 1908-1910 (1999).

9. P. Michler, A. Kiraz, L. Zhang, C. Becher, E. Hu, and A. Imamoglu, "Laser emission from quantum dots in microdisk structures," Appl. Phys. Lett. 77, 184-186 (2000).

10. S. Krishna, S. Raghavan, A. Gray, A. Stintz, and K. Malloy, "Characterization of rapid-thermal-annealed InAs/In.15Ga, 85 As dots-in-well heterostructure using double crystal x-ray diffraction and photoluminescence," Appl. Phys. Lett. 80, 3898-3900 (2002).

11. G. Liu, A. Stintz, H. Li, T. Newell, A. Gray, P. Varangis, K. Malloy, and L. Lester, "The Influence of Quantum-Well Composition on the Performance of Quantum Dot Lasers Using InAs/InGaAs Dots-in-a-Well (DWELL) Structures,” IEEE J. Quan. Elec. 36, 1272-1279 (2000).

12. T. Ide, T. Baba, J. Tatebayashi, S. Iwamoto, T. Nakaoka, and Y. Arakawa, "Lasing characteristics of InAs quantum-dot microdisk from $3 \mathrm{~K}$ to room temperature," Appl. Phys. Lett. 85, 1326-1328 (2004).

13. P. E. Barclay, K. Srinivasan, and O. Painter, "Optical fiber coupling to planar photonic crystal microcavities," submitted for publication, May, 2004 (available at http://www.arxiv.org/abs/physics/0405064). 